\title{
Rotorcraft Acoustic Noise Estimation and Outlier Detection
}

\author{
Johnny $\mathrm{Fu}$, and Xiao-Hua Yu, Member, IEEE
}

\begin{abstract}
This paper focuses on the application of artificial neural networks for rotorcraft acoustic data modeling, prediction, and outlier detection. The original data is recorded by microphones mounted inside a wind tunnel at NASA Ames Research Center, Moffett Field, CA. The experimental data is first acquired in the time-domain as a time history measurement; then the sound pressure level (SPL) that represents the acoustic noise in frequency domain is derived from the time history dataset. In this study, neural networks based models are developed in both time domain and frequency domain. Outlier detection is then performed using modified Z-scores for SPL data to find test points that are statistically inconsistent with the neural network model. Satisfactory computer simulation results are obtained.
\end{abstract}

\section{INTRODUCTION}

Wind tunnel acoustic tests of rotorcraft can be conducted to determine the conditions under which a rotorcraft generates the greatest amount of noise. If pilots are given enough information to avoid these flight conditions, rotorcraft acoustic noise could be reduced in actual flight. This in turn may help rotorcrafts gain greater acceptance in use by the general public. The specific type of noise that rotorcraft researchers are generally interested in for noise reduction is the blade-vortex interaction noise (BVI). This is the noise that occurs when a rotorcraft blade passes through the aerodynamic wake of previous blades, and is the noise that is of the greatest concern for both military and civilian use [1]. Successful modeling and prediction of BVI noise levels may allow for the development of future flight systems that actively control the emission of BVI noise or alert rotorcraft pilots when BVI noise levels are excessively high in noise-sensitive areas or trajectories. However, due to the highly nonlinear relationship between flight conditions and BVI noise level, the closed-form solution is very difficult to obtain with aerodynamics and acoustic analysis.

It is well known that artificial neural networks can be used to perform tasks such as machine learning, association, classification, generalization and optimization [2] [3]. An artificial neural network (ANN) is composed of many nonlinear adaptive processing elements and is capable of approximating any function under certain conditions. ANN has been widely used in the field of system identification, adaptive control, and statistical modeling. There have been

Johnny $\mathrm{Fu}$ was with the Department of Electrical Engineering, California Polytechnic State University, San Luis Obispo, USA. He is now with Sierra Lobo, Inc., Moffett Field, CA, USA.

Xiao-Hua $\mathrm{Yu}$ is with the Department of Electrical Engineering, California Polytechnic State University, San Luis Obispo, CA 93407, USA (e-mail: xhyu@ee.calpoly.edu). some developments in the past several years in the application of neural networks for wind tunnel experiments, such as [4], [5], and [6]. Kottapalli and Kitaplioglu investigated BVI data quality analysis and prediction for the XV-15 tilt-rotorcraft using backpropagation and radial basis function neural networks [4]. Lo and Schimtz demonstrated the prediction of BVI sound pressure levels using neural networks and the Higher Harmonic Control Aeroacoustics Rotor Test (HART) database [5]. Two parameters that are related with flight conditions, including rotor tip-path-plane angle and advance ratio, are considered. Holger et al. used neural networks to model data time histories gathered from in-flight tests conducted by Eurocopter Deutschland on the BO 105 helicopter [6]. The BVI noise is modeled as a function of phase angles sent to the helicopter's individual blade controller.

In this research, feedforward neural networks will be employed to model the acoustic data gathered from a wind tunnel test of a 3-bladed rotorcraft at NASA Ames Research Center. The relationship between the BVI noise and multiple flight conditions are considered here, including the rotor thrust, the advance ratio, the angle of attack of rotor, and the distance between the microphone and test rotor. Two typical situations are considered here, i.e., a high BVI noise case and a low BVI noise case. Different training methods are applied and compared, including backpropagation and the extended Kalman filter algorithm. The trained neural networks in this study is then be used to predict BVI noise levels not included in the training dataset. This prediction test will demonstrate a trained neural network's ability to estimate/interpolate data that was not tested in the wind tunnel. To detect outliers in the wind tunnel data, the median-based modified Z-score method is employed. An outlier is defined as the data point that is statistically inconsistent with the expectations about this point based on the model derived using the data set [7]. Points that deviate greatly from the model could indicate a fault in the hardware data acquisition system or present new information about the BVI noise level data not yet incorporated into the neural network model. Comparing with the conventional method which is based on the average value of the dataset and may be heavily influenced by the presence of outliers, the modified Z-score method is more robust and thus more appropriate for outlier detection.

This paper is organized as follows. First, a brief description on the wind tunnel test setup and data collection is given in section 2. In Section 3, the neural network based model in time-domain is developed, with comparison on different training algorithms. Section 4 concentrates on the 
model of BVI noise level in frequency-domain. The results from a study to identify outliers in the dataset are presented in Section 5. Finally, a conclusion is presented in Section 6.

\section{DATA COLLECTION AND TEST SETUP}

The experimental data used in this study comes from a wind tunnel test of a 3-bladed rotorcraft conducted in the 80 x 120-foot wind tunnel at NASA Ames Research Center at Moffett Field, CA (Fig. 1). The purpose of this test was to determine the relationship between the acoustic noise level and different flight conditions.

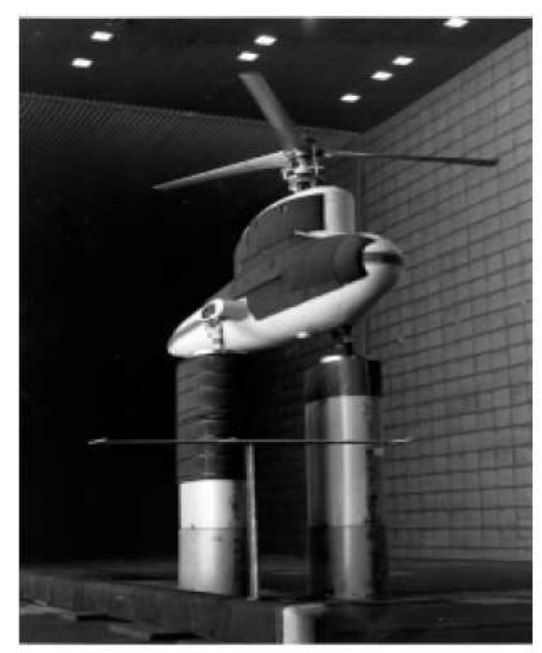

Figure 1. The wind tunnel test setup

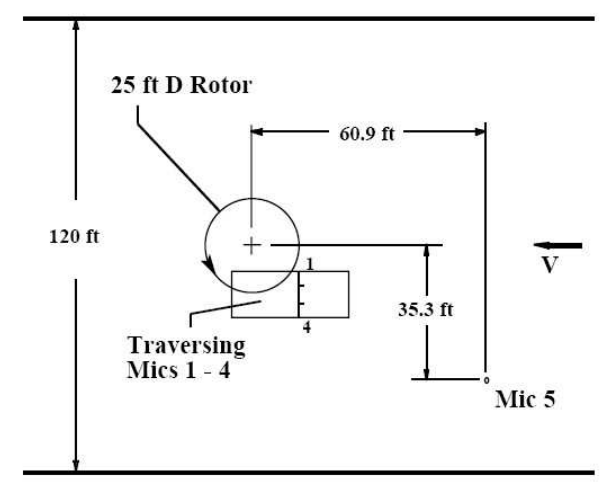

Figure 2. Placement of microphones in wind tunnel experiment

The acoustic data is acquired in the wind tunnel by microphones mounted in strategic locations that are estimated to be the locations of maximum noise relative to the location of the test rotor. Several of these microphones are also mounted on a traverse that allows it to be moved horizontally in the wind tunnel. Individual data points are characterized by a set of parameters, including the rotor thrust $\mathrm{C}_{\mathrm{T}} / \sigma$, a measure of the rotor's lift capability; the advance ratio $\mu$, which is the ratio of true forward airspeed over the wind speed at rotor tip; the rotor tip-path-plane angle $\alpha_{\text {TPP, }}$ also commonly known as the angle of attack; and finally, the distance (measured in inches) between the microphone and test rotor $\mathrm{x}_{\mathrm{m}}$ [4]. Figure 2 shows an example of the placement of microphones relative to a test rotor in a wind tunnel experimental setup.

\section{NEURAL NETWORK BASED MODEL IN TIME-DOMAIN}

A block diagram for neural network based system identification is shown in Fig. 3. A training data set which contains input and desired output (target) is provided; then the weights of the neural network are adjusted to minimize a performance index until it is less than a pre-set threshold.

Mean-square-error (MSE) is the most commonly used criterion and is defined as:

$$
J=\frac{1}{N K}\left[\frac{1}{2} \sum_{n=1}^{N} \sum_{k=1}^{K}\left(z_{k n} \quad d_{k n}\right)^{2}\right]
$$

where $\mathrm{N}$ is the number of input/output pairs in the training set and $\mathrm{K}$ is the number of outputs of the neural network; $\mathrm{Z}_{\mathrm{kn}}$ is the $\mathrm{k}^{\text {th }}$ network output for the $\mathrm{n}^{\text {th }}$ input/output pair in the training set; $d_{k n}$ represents the $k^{\text {th }}$ desired (target) output for the $n^{\text {th }}$ input/output pair. When this performance function is at a minimum, the output of a neural network fits the training data as closely as possible.

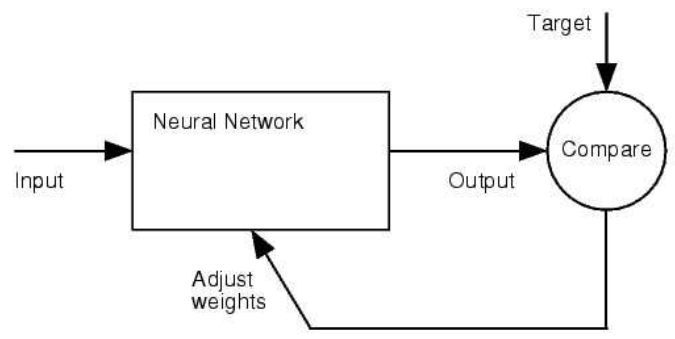

Figure 3. The neural network based system identification

In this research, three different training algorithms are considered. The first one is a multi-layer ADALINE (adaptive linear neuron) neural network trained by LMS (least-mean-square) algorithm. The neural network contains three neurons in the input layer for previous time history values and one output for the BVI noise. All the neurons are linear and there is no hidden layer for this neural network. For the other two neural networks, trained by backpropagation and Kalman filter algorithm respectively, each of them is constructed with a hidden layer of 10 nonlinear neurons (sigmoid activation function) and a linear output neuron.

The weight of neural network can be adjusted using:

$$
w_{m}=w_{m 1}+w_{m}
$$


where $\mathrm{m}$ is the number of training iteration.

Learning in back-propagation is performed in two steps, i.e., the forward pass and the backward pass. In the latter, the error signal (i.e., the difference between the neural network output and the target) is feedback from the output to the input, layer by layer, and all the weights are adjusted in proportion to this error, i.e.,

$$
w_{m}=\frac{J}{W}
$$

where is called the learning/training rate; $J$ is the objective function defined in Eq. (1) and $\mathrm{W}$ is the weight matrix of the neural network. For sigmoid function, we have:

$$
g(u)=\frac{1}{1+e^{u}}
$$

where $g$ is the output of the nonlinear neuron and

$$
u=W X
$$

with weight matrix $\mathrm{W}$ and input to the neuron $\mathrm{X}$ (both vectors). Therefore,

$$
\frac{J}{W}=\left(\begin{array}{ll}
z & d
\end{array}\right) u\left(\begin{array}{ll}
1 & u
\end{array}\right) X
$$

For the sake of simplicity, the index of neuron is not included in the above formula.

It is well known that the extended Kalman filter algorithm can be used to estimate the state of a nonlinear system. If the neural network is considered as a nonlinear system, then the weights can be updated through the Kalman gain $\mathrm{K}$ in each training iteration $\mathrm{m}$ :

$$
\begin{aligned}
& w_{m}=w_{m 1}+K_{m}\left[\begin{array}{ll}
z & d
\end{array}\right] \\
& K_{m+1}=P_{m} H_{m}\left[R+H_{m}^{T} P_{m} H_{m}\right]^{1} \\
& P_{m+1}=P_{m} \quad K_{m} H_{m}^{T} P_{m}
\end{aligned}
$$

where $\mathrm{R}$ is the covariance of the identification error. Again for the sake of simplicity, the index of neuron is not included in the above formula.

In the wind tunnel test, the acoustic level data was recorded at the following wind tunnel conditions for a 3bladed rotorcraft: $\mathrm{C}_{\mathrm{T}} / \sigma=0.04, \mu=0.1, \alpha_{\mathrm{TPP}}=-10^{\circ}$, and $\mathrm{x}_{\mathrm{m}}=$ 364 inches. Three neural networks with different training algorithms are used here for simulation (i.e., the ADALINE network with learning rate set at 0.5 , the back-propagation trained neural network with 10 hidden neurons and a learning rate of 0.5 , and the Kalman filter trained neural network with 10 hidden neurons and an $\mathrm{S}$ value (error covariance) of $510^{4}$ ). The simulation results are shown in Fig. 4. It is shown that the extended Kalman filter training algorithm outperforms the other two networks, with the minimum amount time delay and the lowest MSE (meansquare-error).

The high BVI case of the 3-bladed rotorcraft is also studied in this research. The measurement data is taken under the following test conditions: $\mathrm{C}_{\mathrm{T}} / \sigma=0.09, \mu=0.19$,
$\alpha_{\mathrm{TPP}}=4^{\circ}$, and $\mathrm{x}_{\mathrm{m}}=-70$ inches. From the simulation result shown in Fig. 5, we find that the extended Kalman filter training algorithm again yields the best overall performance with MSE being only $25 \%$ of the MSE for the backpropagation algorithm. The ADALINE algorithm has the worst performance and produces larger errors on the BVI peaks.

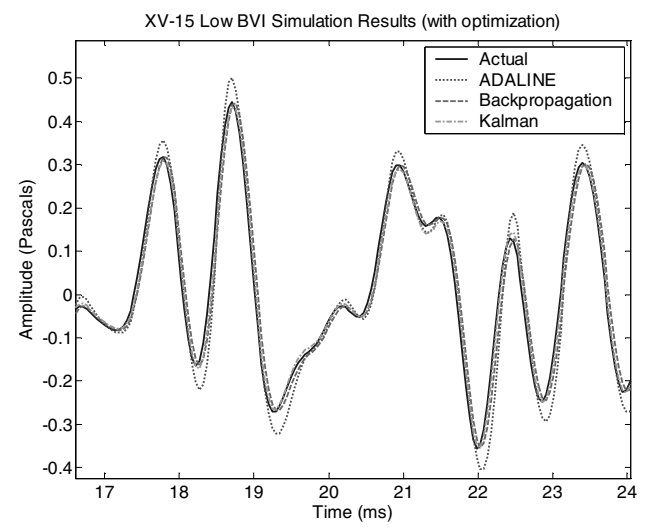

Figure 4. Simulation results on 3-bladed rotorcraft (low-BVI case)

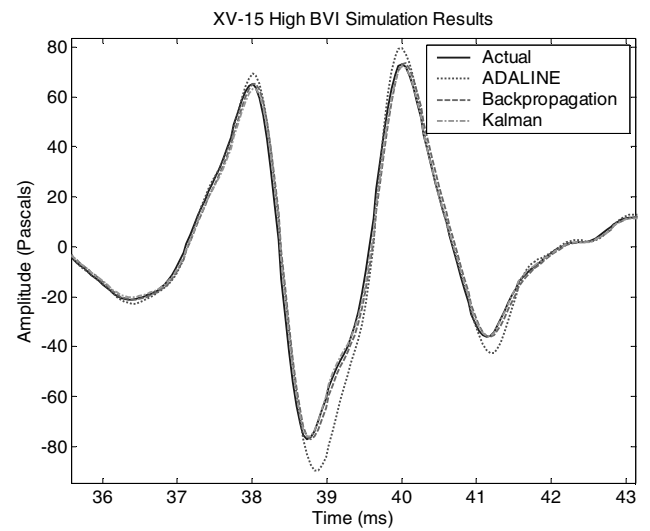

Figure 5. Simulation results on 3-bladed rotorcraft (high-BVI case)

The simulation results with different training algorithms are summarized in table 1 , where the percentages of errors are shown for each situation:

TABLE I

SIMULATION RESULTS TABLE FOR DIFFERENT TRAINING ALGORITHMS

\begin{tabular}{|c|c|c|}
\hline Algorithm & Low BVI & High BVI \\
\hline ADALINE & $14.25 \%$ & $13.45 \%$ \\
\hline Backpropagation & $7.45 \%$ & $7.04 \%$ \\
\hline Kalman & $2.1 \%$ & $2.95 \%$ \\
\hline
\end{tabular}

\section{BVI NOISE LEVEL ESTIMATION}


The sound pressure level metric provides a convenient way for rotorcraft researchers to study the overall BVI acoustic noise for a given set of test parameters. To obtain a single-value representing the overall BVI noise level from one rotor revolution, the time-domain data is transformed into the frequency domain using a FFT. The resulting power spectrum is integrated and converted from units of Pascals ${ }^{2}$ to units of $\mathrm{dB}$ to obtain a BVI noise level value that characterizes the acoustic noise from one revolution of the rotor. A digital bandpass filter is applied to highlight the BVI frequencies.

The whole dataset contains about 1000 total BVI sound pressure level measurements for 4 microphones. The database was divided into three approximately equal datasets for training, validation, and testing. The inputs to the neural network include $\mathrm{C}_{\mathrm{T}} / \sigma, \mu, \alpha_{\mathrm{TPP}}$, the microphone number, and the traverse position (in inches). The neural network contains 10 hidden neurons, and is trained off-line. To speed up the training, the extended-bar-delta-bar adaptive learning rate algorithm is used:

$$
w_{m}=w_{m 1}(1 \quad)_{m m}
$$

and ${ }_{m}=J / W$

where is the momentum factor (commonly 0.9 for most applications) and ${ }_{m}$ is called the adaptive learning rate. A mean square error of $6 \quad 10^{4}$ is achieved in approximately 10,000 training epochs.

In the studies of BVI sound pressure level, a contour plot which shows the relationship between BVI and the variations of two flight parameters is very important. However, in the case when there are not enough points in the dataset, the contour plot may not display any recognizable pattern due to the lack of measurement. Fig. 6 shows one example when the two flight parameters $\alpha_{\mathrm{TPP}}$ (the rotor tippath-plane angle, or the angle of attack) and the advance ratio $\mu$ at the rotor tip.

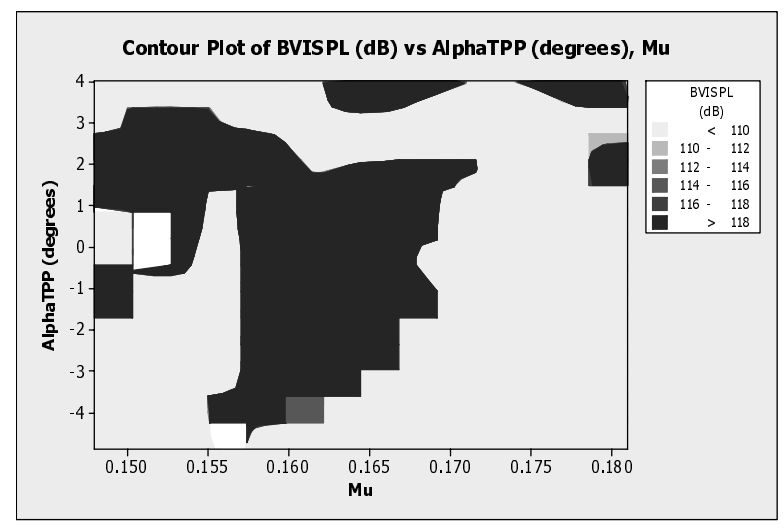

Figure 6. Relationship between the BVI noise level vs. $\mu$ and $\alpha_{\text {TPP }}$
Using the trained neural network, it is possible to interpolate the incomplete dataset with the points predicted by the neural network. A complete dataset can then be constructed and a contour plot which is more visible can be generated (Fig. 7).

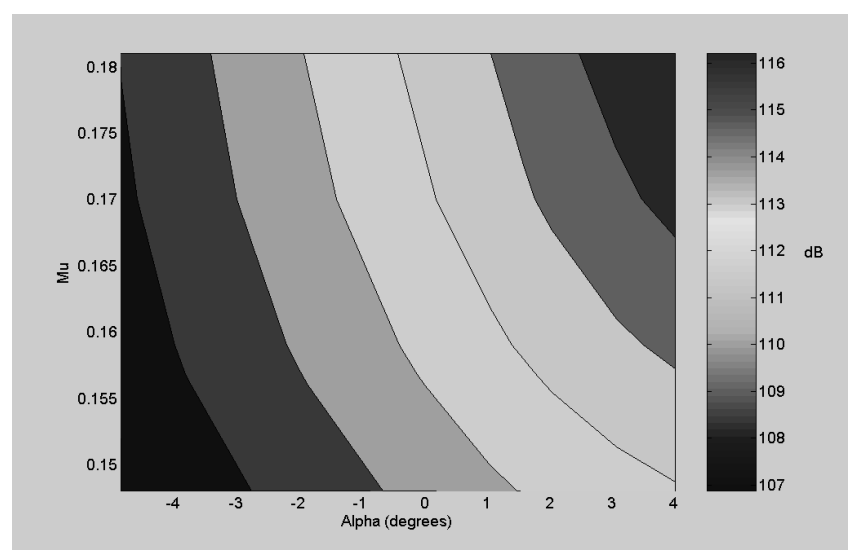

Figure 7. Relationship between the BVI noise level vs. $\mu$ and $\alpha_{T P P}$ (reconstructed with neural network model)

\section{OUTLIER DETECTION}

The conventional method for outlier detection is based on the average value of the dataset and may be heavily influenced by the presence of outliers. In this research, the robust modified Z-score method is used to find points where there the residual between the estimated neural network output and the recorded BVI noise level are outside the range of residuals for the bulk of the data. To determine this range of residuals, a benchmark median absolute deviation from the median residual value is calculated (M.A.D.) using the prediction residuals of the trained neural network. The equation for this M.A.D. value is shown in Eq. (10) where the residuals are contained in a set $\{e\}, e_{i}$ is the residual for the $\mathrm{i}^{\text {th }}$ data point, and $\tilde{e}$ is the median residual:

$$
\text { M.A.D. }=\text { median }\left\{\left|e_{i} \tilde{e}\right|\right\}
$$

Once the benchmark M.A.D. is calculated, a modified Z-score (M) is calculated for each prediction residual using Eq. (11). Here, the scaling factor 0.6745 is used to normalize the expected value of the M.A.D. so that it is equal to the standard deviation in a normal distribution.

$$
M_{i}=\frac{0.6745\left(e_{i} \quad \tilde{e}\right)}{M A D}
$$

The absolute value of the modified Z-score for each point is compared to the M.A.D. to determine whether it is an outlier point or not. When $\left|M_{i}\right|>\quad$ (M.A.D.), the $\mathrm{i}^{\text {th }}$ data point is identified as an outlier. When $\psi=3.5$ in normally distributed data, approximately $5 \%$ of the data points are identified as outliers. 
For the BVI noise level data, an analysis of the outliers in the original 309 point testing dataset is conducted. The MAD of the validation residuals was calculated to be 1.57 dB. Using an outlier factor of 3.5, 6 outliers were detected in the data. The residuals from these outlier points ranged from $-5.73 \mathrm{~dB}$ to $6.67 \mathrm{~dB}$. Three of these points came from a common set of input parameters $\left(\mathrm{C}_{\mathrm{T}} / \sigma=0.0754, \mu=0.125\right.$, and $x_{m}=225$ ). Such a finding would indicate the need for closer inspection of the wind tunnel test $\log$ for these points to see if any unusual occurrences were recorded for these data points. Lowering the outlier factor to 2.5 increases the number of outliers detected to 18 points. These points have absolute residuals $>4 \mathrm{~dB}$. It is noticed that several of these outliers were different microphones with the same set of wind tunnel operating parameters. This means that for these data points, the neural network predicted values and the wind tunnel values compared poorly and thus the data for those points require a closer inspection.

\section{CONCLUSION}

In this research, artificial neural networks are successfully employed to estimate BVI noise levels and compared with the actual data gathered from wind tunnel data in both time and frequency domain. The simulation results showed that a trained neural network is able to capture the relationship between the different flight parameters and the corresponding BVI noise level in frequency domain. Outlier detection was performed using modified Z-scores and a benchmark M.A.D. statistic calculated from the neural network prediction residuals. Only a few outliers were detected in the non-training portion of the dataset, indicating that the overall wind tunnel data was consistently recorded.

Future works that may evolve from this study include using the neural network to analyze other sets of rotorcraft data. Other data sets may be analyzed following the methodology of this study for the purposes of modeling, prediction, and outlier detection.

\section{ACKNOWLEDGEMENTS}

The author would like to thank Dr. William Warmbrodt, Cahit Kitaplioglu, and the other members of the Aeromechanics Branch at NASA Ames Research Center for supporting this work. This work was also partially sponsored by the Department of the Navy, Office of Naval Research, under Award \# N00014-05-1-0855.

\section{REFERENCES}

[1] W. Johnson, Helicopter Theory (Princeton, NJ: Princeton University Press, 1980).

[2] A. Zaknich, Neural Network for Intelligent Signal Processing (Singapore: World Scientific, 2003).

[3] R.J. Schalkoff, Artificial Neural Networks (New York: McGraw-Hill, 1997).
[4] C. Kitaplioglu, Blade-Vortex Interaction Noise of a Full-Scale XV-15 Rotor Tested in the NASA Ames 80 - by 120 -Foot Wind Tunnel, NASA Technical Memorandum 208789. Moffett Field: National Aeronautics and Space Administration.

[5] Lo, and Schmitz, F., Model-Based Neural Networks for Rotorcraft Ground Noise Prediction, Proceedings of the $38^{\text {th }}$ AIAA Aerospace Sciences Meeting \& Exhibit, Reno, NV, January 10-13, 2000.

[6] Holger, G., et al. Neural Networks For BVI System Identification, Proceedings of the $29^{\text {th }}$ European Rotorcraft Forum, Friedrichshafen, Germany, September 16-18, 2003.

[7] B. Iglewicz and D.C. Hoaglin, How to Detect and Handle Outliers (Milwaukee, WI: ASQC Quality Press, 1993).

[8] Fu, J., Modeling and analysis of rotorcraft acoustic data with neural networks, M. S. thesis, June 2004. 